\title{
AFFINE SEMIGROUPS
}

\section{BY \\ HASKELL COHEN AND H. S. COLLINS( $\left.{ }^{1}\right)$}

In what follows $X$ and $Y$ are topological vector spaces (Hausdorff) over the reals, and $S$ and $T$ are subsets of $X$ and $Y$ respectively. When $S$ and $T$ are convex, a mapping $f: S \rightarrow T$ is said to be affine if $f$ preserves convex combinations: $x, y \in S, 0 \leqq \alpha \leqq 1$ implies $f(\alpha x+(1-\alpha) y)=\alpha f(x)+(1-\alpha) f(y)$. It is easy to verify (and well known) that if $f$ is affine, then $f$ preserves more general combinations: $f\left(\sum_{i=1}^{n} \alpha_{i} x_{i}\right)=\sum_{i=1}^{n} \alpha_{i} f\left(x_{i}\right)$ whenever $x_{1}, \cdots, x_{n} \in S$, $\alpha_{1}, \cdots, \alpha_{n} \geqq 0, \sum_{i=1}^{n} \alpha_{i}=1$, and $\sum_{i=1}^{n} \alpha_{i} x_{i} \in S$. The set $S$ is said to be an affine semigroup when $S$ is convex; $S$ is a semigroup (relative to some multiplication on $S$ ), and multiplication is separately affine; i.e., for each $x \in S$, the mappings $y \rightarrow x y$ and $y \rightarrow y x$ are affine functions. If, in addition, $S$ is a topological semigroup (relative to the topology inherited from $X$ ), $S$ is an affine topological semigroup.

Such semigroups are often encountered: in Theorem 8 below it is shown that a finite dimensional affine semigroup with unit (identity element) and zero is always a matrix semigroup, and it is clear that every convex set of linear transformations of $X$ into itself which is a semigroup under composition is an affine semigroup. An infinite dimensional affine semigroup of special interest is the semigroup of measures on a compact group [9].

$\$ 1$ of the paper is devoted to some properties of general affine (not necessarily finite dimensional) semigroups, including for the sake of completeness several results due to others. $\$ 2$ gives a complete characterization of all one and two dimensional compact affine topological semigroups and of all one dimensional affine semigroups; all possible multiplications are listed in each of these cases. The final $\$ 3$ includes proofs that the minimal ideal of each one or two dimensional compact affine topological semigroup is convex; a similar statement can be made for three dimensional semigroups with unit, but examples are given which show the theorem is false for dimension three without unit and for dimension $\geqq$ four in general.

1. The first theorem of this section is due to J. G. Wendel (unpublished); its proof is included for the sake of completeness. First, however, recall that the space $X$ is locally convex if there is a basis for open sets at the additive identity of $X$ consisting of convex sets, and an extreme point of convex $S$ is a

Presented to the Society, August 30, 1957 under the titles Admissible mobs, I. Preliminary report, and Convexity of the kernel in admissible mobs; received by the editors July 25, 1958.

(1) This work was supported by the United States Air Force through the Air Force Office of Scientific Research, Air Research and Development Command, under Contract AF 18(603)-89. 
point $x$ which is interior to no line segment of $S$ : if $x=\alpha y+(1-\alpha) z$, with $0<\alpha<1, y, z \in S$, then $x=y=z$. It is well known that extreme points of a compact convex subset $S$ of a locally convex space exist, and in fact, the smallest closed convex set containing the set of extreme points of $S$ is $S$ itself [4].

THEOREM 1 (WENDEL). If $S$ is a compact affine topological semigroup with unit, and $X$ is locally convex, then every element of $S$ with inverse is an extreme point of $S$.

Proof. Denote by $u$ the unit of $S$ and let $u=\alpha x+(1-\alpha) y$, where $x, y \in S$, $0<\alpha<1$. If $q$ is an extreme point of $S$, then $q=q u=\alpha q x+(1-\alpha) q y$, so $q=q x$ $=q y$. A similar statement holds for convex combinations of extreme points of $S$ : if $z=\sum_{i=1}^{n} \alpha_{i} q_{i}$, with $\sum_{i=1}^{n} \alpha_{i}=1, \alpha_{1}, \cdots, \alpha_{n} \geqq 0$, and $q_{1}, \cdots, q_{n}$ extreme points, then $z x=\sum_{i=1}^{n} \alpha_{i} q_{i} x=\sum_{i=1}^{n} \alpha_{i} q_{i} y=z y$. But then, the Krein-Milman theorem shows that $u$ is the limit of a net [3] $\left\{x_{n}\right\}$, where $x_{n} x=x_{n} y$, hence $x=u x=\left(\lim _{n} x_{n}\right) x=\lim _{n}\left(x_{n} x\right)=\lim _{n}\left(x_{n} y\right)=\left(\lim _{n} x_{n}\right) y=u y=y$. Thus, $u$ is an extreme point. Finally, if $t \in S$ has an inverse and $t=\alpha x+(1-\alpha) y, x, y \in S$, $0<\alpha<1$, then $u=t^{-1} t=\alpha t^{-1} x+(1-\alpha) t^{-1} y$ implies $t^{-1} x=t^{-1} y$, so $x=y$, and $t$ is an extreme point.

It perhaps should be remarked that Wendel assumed $S$ was a subset of a topological algebra, but it is clear that his proof of the above theorem requires only a multiplication of the proper sort on $S$.

The proof of the next theorem can be made to depend on the fixed point theorem of Tychonoff: every continuous mapping of a convex compact subset of a locally convex space has a fixed point. However, all that is required is the fact that every continuous affine mapping of a convex compact subset of a locally convex space has a fixed point; a simple proof of the latter theorem was given by Nikaido in [5].

Theorem 2. Let $X$ be locally convex, $T \subset X$ be a compact affine topological semigroup with unit, $G$ be a closed subgroup of $T$, and $S$ be the closed convex hull of $G$. Then there exists points $\hat{s} \in S, \hat{g} \in G$ such that $\hat{s} S \hat{g}=\{\hat{s} \hat{g}\}$.

Proof. Let $A=\left\{a_{1}, \cdots, a_{m}\right\} \subset G, B=\left\{f_{1}, \cdots, f_{n}\right\} \subset X^{*}$, the space of continuous linear functions on $X$. Define $F: X \rightarrow$ Euclidean $n$-space by $F(x)$ $=\left(f_{1}(x), \cdots, f_{n}(x)\right)$. Then $F$ is continuous and linear. Now the mapping $s \rightarrow m^{-1} \sum_{i=1}^{m} s a_{i}$ of $S$ into itself is affine and continuous, so by Nikaido's theorem there exists $s_{0}$ in $S$ such that $s_{0}=m^{-1} \sum_{i=1}^{m} s_{0} a_{i}$, and hence $s_{0} g$ $=m^{-1} \sum_{i=1}^{m} s_{0} a_{i} g$, all $g \in G$. But then $F\left(s_{0} g\right)=m^{-1} \sum_{i=1}^{m} F\left(s_{0} a_{i} g\right)$, all $g \in G$, so the compactness of $G$ implies there exists $g_{0}$ in $G$ such that $\left\|F\left(s_{0} g_{0}\right)\right\|$ $=\max \left\{\left\|F\left(s_{0} g\right)\right\|: g \in G\right\}$. Then $\left\|F\left(s_{0} g_{0}\right)\right\|=\left\|m^{-1} \sum_{i=1}^{m} F\left(s_{0} a_{i} g_{0}\right)\right\|$ $\leqq m^{-1} \sum_{i=1}^{m}\left\|F\left(s_{0} a_{i} g_{0}\right)\right\|$, and $\left\|F\left(s_{0} g_{0}\right)\right\| \geqq\left\|F\left(s_{0} a_{i} g_{0}\right)\right\|$ for all $i$, by the choice of $g_{0}$ and the fact that $a_{i} g_{0} \in G$; thus $\left\|F\left(s_{0} g_{0}\right)\right\|=\left\|F\left(s_{0} a_{i} g_{0}\right)\right\|, i=1, \cdots, m$. The latter fact together with $F\left(s_{0} g_{0}\right)=m^{-1} \sum_{i=1}^{m} F\left(s_{0} a_{i} g_{0}\right)$ implies $F\left(s_{0} g_{0}\right)$ 
$=F\left(s_{0} a_{i} g_{0}\right), i=1,2, \cdots, m$. An obvious compactness argument implies there exists $(\xi, \hat{g}) \in \bigcap\left\{N(A, B):\right.$ A finite $\subset G, B$ finite $\left.\subset X^{*}\right\}$, where $N(A, B)$ $=\{(s, g): s \in S, g \in G$, and $f(s g)=f(s a g)$, all $a \in A, f \in B\}$, and it follows that $f(\hat{s} \hat{g})=f(\hat{s} a \hat{g})$, each $a \in G$ and all $f \in X^{*}$. However, $X^{*}$ separates points of $X$, and in particular, of $S$, so that $\hat{s} \hat{g}=\hat{s} a \hat{g}$, all $a \in G$; i.e., $\{\hat{s} \hat{g}\}=\hat{s} G \hat{g}$. But then since $S$ is the closed convex hull of $G$, it is also true that $\{\hat{s} \hat{g}\}=s S \hat{g}$.

COROLlaRY. If $S$ is a compact affine topological semigroup and if every extreme point of $S$ has an inverse, then $S$ has a zero.

Proof. Let $G$ be the set of elements with inverse; $G$ is known to be a compact group [7], and the hypotheses imply that the closed convex hull of $G$ is $S$. An application of Theorem 2 yields $s_{1} \in S, g_{1} \in G$ such that $\left\{s_{1} g_{1}\right\}=s_{1} S g_{1}$, and an application of the left-right dual of Theorem 2 yields $s_{2} \in S, g_{2} \in G$ such that $\left\{g_{2} s_{2}\right\}=g_{2} S s_{2}$. Since $g_{1}, g_{2}$ have inverses, $\left\{s_{1}\right\}=s_{1} S$ and $\left\{s_{2}\right\}=S s_{2}$, i.e.; $s_{1}$ is a left zero and $s_{2}$ is a right zero. It is then clear that $s_{2} s_{1}$ is a zero.

The preceding corollary was proved by Peck in [6]. However, again it was assumed there that $G$ was a subset of a topological algebra, while the above proof shows that only a local assumption of multiplication is required. The idea of the proof of Theorem 2 is basically the same as that used by Nikaido in his proof of the invariant mean-value theorem [5];

Minimal ideals and idempotents play a crucial role in this paper, and the reader is referred to [8] and [1] for the pertinent facts concerning them. It is shown there that any compact topological semigroup $S$ has minimal left ideals, minimal right ideals, and a unique minimal two-sided ideal $K$, called the kernel, that all of these are (topologically) closed, that $K$ is the union of the minimal left ideals and also of the minimal right ideals, and finally, that the intersection of any minimal right and minimal left ideal is a group. As a consequence of the last result, $S$ always contains an idempotent; in the affine case this follows easily from the Tychonoff fixed-point theorem, for the mapping $x \rightarrow x^{2}$ of $S$ into itself has a fixed point.

The next theorem contains some of the obvious information concerning the ideals; a more detailed analysis is presented in the final section three.

TheOREM 3. Let $S$ be a compact affine topological semigroup with $X$ locally convex. Then (a) each minimal lejt or right ideal of $S$ is convex, (b) $x \in K$ if and only if $x S x=\{x\}$; in particular, each point of the kernel is an idempotent.

Proof. (a) If $L$ is a minimal left ideal and $e \in L$, then $L=S e$, by minimality. The mapping $x \rightarrow x e$ of $S$ onto $L$ is affine and its image is convex, since an affine function clearly preserves convexity. A similar proof holds for minimal right ideals.

(b) If $x \in K$, there exist unique $L$ and $R$ (minimal left and right ideals respectively) for which $x \in L \cap R$, and it was shown in [1] that $I \cap R=e S \cap S e$ $=e S e$, if $e$ denotes the unit of the group $e S e$. Note that the mapping $y \rightarrow e y e$ 
is affine with image $e S e$, so $e S e$ is convex. It is also compact, and so is a compact affine group, with unit $e$. It then follows from Theorem 1 that $e S e$ is a point, so that $e=x, x$ is idempotent, and $\{x\}=x S x$. Conversely, if $\{x\}=x S x$, fix $e \in K$; then $x=x e x \in K$, the latter since $K$ is an ideal.

The fact that $e S e$ is a point also follows directly from the Tychonoff theorem, for by that theorem $e S e$ is a group with the fixed point property; such groups must be points.

A linear manifold (manifold, affine subspace) $M$ of $X$ is a translate of some linear subspace $N$. It is well known that under these circumstances, $N+a=M$ for every $a \in M$. The manifold generated by a nonvoid subset $A$ of $X$ is the intersection of all manifolds containing $A$; the notation $M(A)$ is used throughout this paper. $M(A)$ is a manifold, and is known to be the set of all points $\sum_{i=1}^{n} \alpha_{i} x_{i}+\left(1-\sum_{i=1}^{n} \alpha_{i}\right) a$, for any $a \in A$, where $n$ is a positive integer, and $x_{1}, \cdots, x_{n} \in A$. These sets play an important part in sections two and three, and their use is also required for the next several theorems.

THEOREM 4. If $S$ is a compact affine topological semigroup and $L$ is a minimal left ideal, then $M(L) \cap S=L$ (dually for minimal right ideals).

Proof. Since $L \subset M(L)$, it is trivial that $L \subset M(L) \cap S$. For the reverse inclusion, fix $e \in L$ and let $x \in M(L) \cap S$. Then $x=\sum_{i=1}^{n} \alpha_{i} x_{i}+\left(1-\sum_{i=1}^{n} \alpha_{i}\right) e$, for some $n$ and $x_{1}, \cdots, x_{n} \in L$. But then (since $e$ is a right unit for $L$ ) $x e$ $=\sum_{i=1}^{n} \alpha_{i} x_{i} e+\left(1-\sum_{i=1}^{n} \alpha_{i}\right) e^{2}=\sum_{i=1}^{n} \alpha_{i} x_{i}+\left(1-\sum_{i=1}^{n} \alpha_{i}\right) e=x$; hence $x=x e$ $\in S e=L$.

THEOREM 5. Let $S$ be a compact affine topological semigroup with kernel $K$ and $E$ its set of idempotents. Denote by $H$ the union of all the sets $H_{\theta}$, for $e \in E \backslash K$, where $H_{e}$ is the maximal subgroup containing $e$. Then $H$ is contained in the boundary of $S$.

Proof. First let $e \in E \backslash K$, and fix $f \in K$. Then $e f e \in K$, so $e f e \in E$ and $e f e \neq e$. Let $M$ denote the manifold generated by $e$ and $e f e$; it is easily verified that $M$ is an affine semigroup with $e$ as unit and $e f e$ as zero and that the line segment $T=M \cap S$ is a compact affine topological semigroup. Now $e$ is an endpoint of $T$ : if not, there exist $a \in S$ and $\alpha>1$ such that $a=\alpha e+(1-\alpha)$ efe, and it follows that $a^{n}=\alpha^{n} e+\left(1-\alpha^{n}\right) e f e$, for all $n$. Since $e \neq e f e$, there exists $F \in X^{*}$ such that $F(e) \neq F(e f e)$. Now if $F(e f e)=0,\left\{F\left(a^{n}\right)\right\}=\left\{\alpha^{n} F(e)\right\}$, an unbounded set of real numbers. If $F(e f e) \neq 0$, let $G=F / F(e f e) \in X^{*}$; then $\left\{G\left(a^{n}\right)\right\}=\left\{\alpha^{n}[G(e)-1]+1\right\}$, again an unbounded sequence of reals. Thus, $S$ is unbounded, contradicting the compactness of $S$. Hence $e$ is an end-point of $T$, and it follows that $e$ is a boundary point: if not, $e \in S^{0}$ (the interior of $S$ ), and there exist $x, y \in T$ and $0<\alpha<1$ such that $e=\alpha x+(1-\alpha) y$, contradicting the fact that $e$ is an end-point of $T$.

Now, let $x \in H$, so that there exists $e \in E \backslash K$ such that $x \in H_{e}$. If $H_{e}=\{e\}$, then $x=e$ is a boundary point. If $H_{e} \neq\{e\}$ and $T$ is the closed convex hull of 
$H_{\bullet}$, then $T$ is a compact affine topological sub-semigroup of $S$, and the corollary to Theorem 2 shows there is a zero $z$ for $T$, with $z \notin H_{e}$. Now one can proceed as above, by showing that $x$ is an end-point in $S$ of the manifold through $z$ and $x$, and hence is a boundary point of $S$ : if $x$ is not an end-point there exist $a \in S$ and $\alpha>1$ such that $a=\alpha x+(1-\alpha) z$, and hence $a x^{-1}=\alpha e+(1-\alpha) z$. But then, exactly as above, since $e$ is the unit of $M(\{e, z\})$ and $z$ its zero, one knows that $e$ is an end-point in $S$ of $M(\{e, z\})$, so $\alpha \leqq 1$, a contradiction.

The previous theorem should be compared with Theorem 1; that theorem gives the result that $H_{u}$ is contained in the set of extreme points if $u$ is the unit (and so is clearly contained in the boundary), while Theorem $\mathbf{5}$ says for every idempotent $e$ not in the kernel that $H_{e}$ is contained in the boundary (examples yield many $H_{e}$ 's which are not contained in the set of extreme points).

Two affine semigroups $S$ and $T$ are said to be equivalent if there exists a 1-1 affine function $f$ of $S$ onto $T$ which at the same time is an isomorphism; if $S$ and $T$ are topological semigroups as well $f$ is required to be bi-continuous. Motivation for this definition of equivalence is found in the following theorem and in the fact that each finite dimensional affine semigroup (see $\$ 2$ ) is equivalent under such a mapping to an affine semigroup of Euclidean $n$-space.

THEOREM 6. Let $S$ be an affine semigroup and $f$ a 1-1 affine mapping on $S$ to $X$, with $T=f(S)$. Then $T$ is an affine semigroup relative to the multiplication induced on $T$ from $S$ by $f$. If, in addition, $S$ is a topological semigroup and $f$ is bi-continuous, then $T$ is also an affine topological semigroup relative to this multiplication.

Proof. Since $f$ is affine and $S$ convex, it is clear that $T$ is convex. Now, for $p$ and $q \in T$, define $p q=f\left[f^{-1}(p) \cdot f^{-1}(q)\right]$. It is clear that this product makes $T$ into a semigroup, and all that should be checked is that $T$ is affine. If $p, q$, $r \in T, 0 \leqq \alpha \leqq 1$, then $p[\alpha q+(1-\alpha) r]=f\left[f^{-1}(p) \cdot f^{-1}\{\alpha q+(1-\alpha) r\}\right]$, and the latter $=f\left[f^{-1}(p) \cdot\left\{\alpha f^{-1}(q)+(1-\alpha) f^{-1}(r)\right\}\right]$ since $f^{-1}$ is also affine. But then this is $\left.f\left[\alpha f^{-1}(p) \cdot f^{-1}(q)+(1-\alpha) f^{-1}(p) \cdot f^{-1}(r)\right)\right]$ since $S$ is affine, and this in turn is $\alpha p q+(1-\alpha) p r$, since $f$ is affine. This multiplication is clearly continuous if $S$ is topological and $f$ is a homeomorphism.

Under certain conditions the multiplication on an affine semigroup $S$ can be extended to all of $M(S)$, and this fact is of importance in $\S \S 2$ and 3 . The next few theorems give information bearing on this extension problem. Preliminary to this, however, we need the notion of dimension (and it is the usual linear dimension). The dimension of a convex set $S$ is the cardinal number of a basis of $M(S)-a$, for any fixed $a \in S$. This definition is meaningful, since $M(S)-a$ is a linear space (and so has a basis), and $M(S)-a=M(S)-b$, for any $a, b \in S$. In case $S$ is finite dimensional, say of dimension $n$, one sees easily that an equivalent formulation is that every element $x$ of $M(S)$ be uniquely expressible as $x=\sum_{i=1}^{n} \alpha_{i} x_{i}+\left(1-\sum_{i=1}^{n} \alpha_{1}\right) a$, for any fixed set 
$\left\{x_{1}, x_{2}, \cdots, x_{n}, a\right\} \subset S$ for which $\left\{x_{1}-a, \cdots, x_{n}-a\right\}$ is linearly independent. Such a set $\left\{x_{1}, x_{2}, \cdots, x_{n}, a\right\} \subset S$ is called an affine basis for $M(S)$.

Theorem 7. Let $S$ be an $n$-dimensional affine semigroup, and $M(S)$ be the manifold generated by $S$. Then the multiplication on $S$ is uniquely extendable over $M(S)$, and relative to this multiplication $M(S)$ becomes an affine semigroup.

Proof. Let $\left\{x_{1}, \cdots, x_{n}, x_{n+1}\right\} \subset S$ be an affine basis for $M(S), x, y \in \dot{M}(S)$, and $x=\sum_{i=1}^{n+1} \alpha_{i} x_{i}, \quad y=\sum_{j=1}^{n+1} \beta_{j} x_{j}, \quad \sum_{i=1}^{n=1} \alpha_{i}=1, \quad \sum_{j=1}^{n+1} \beta_{j}=1$, and define $x y=\sum_{1 \leq i, j \leq n+1} \alpha_{i} \beta_{j} x_{i} x_{j}$. Now this product clearly depends on the choice of basis; it is, however, separately affine on $M(S) \times M(S)$ to $M(S) \times M(S)$ and agrees with the given multiplication when restricted to $S \times S$. Similar statements hold if a product is defined by means of a different affine basis, and the uniqueness of product then follows from the fact that any two separately affine functions on $M(S) \times M(S)$ to $M(S) \times M(S)$ which agree on $S \times S$ are identical. Finally, the associativity of the multiplication on $M(S)$ follows directly from the facts that the multiplication is separately affine on $M(S)$ and is associative when restricted to $S$.

THEOREM 8. Every finite dimensional affine semigroup $S$ with left or right unit is equivalent to a semigroup (under composition) of affine transformations of $M(S)$. In particular, if $S$ also has either a left or right zero, then $S$ is cquivalent to a semigroup of matrices, each having real entries.

Proof. Let $\left\{x_{1}, \cdots, x_{n}, x_{n+1}\right\}$ be a fixed affine basis for $M(S)$ and if $x \in S$, define $l_{x}: M(S) \rightarrow M(S)$ by $l_{x}(y)=x y$, for $y \in M(S)$, where the product $x y$ is justified by Theorem 7. The mapping $l_{x}$ is clearly affine, and if $l_{x}=l_{y}$ and $u$ is a right unit, then $x=x u=l_{x}(u)=l_{y}(u)=y u=y$; i.e., the mapping $x \rightarrow l_{x}$ embeds $S$ in a 1-1 fashion into a set of affine functions of $M(S) \rightarrow M(S)$. Now if $x, y \in S$ and $\alpha+\beta=1$, one sees that $l_{x y}(z)=(x y) z=x(y z)=l_{x}\left(l_{y}(z)\right)$ $=\left(l_{x} \cdot l_{y}\right)(z)$ and $l_{\alpha x+\beta y}(z)=(\alpha x+\beta y) z=\alpha x z+\beta y z=\alpha l_{x}(z)+\beta l_{y}(z)$, for all $z \in M(S)$; i.e., $x \rightarrow l_{x}$ is a homomorphism between $S$ and its image and is affine. In case $S$ has a left unit the procedure just outlined can be used, with left multiplications being replaced by right ones.

If $S$ has a right zero $e$, let $T=S-e$, and $\theta=$ the additive unit of $X$. Then $T$ becomes (Theorem 6) an affine semigroup with right or left unit and with $\theta$ as right zero, and is equivalent to $S$. By the first part of this theorem, $T$ is equivalent to a semigroup $T_{n}$ of affine transformations of the $n$-dimensional vector space $L=M(T)$. But note that if $f: L \rightarrow L$ is the affine mapping $l_{x}$, where $x \in T$, then $y, z \in M(T), \alpha, \beta$ real $\Rightarrow f(\alpha y+\beta z)=l_{x}(\alpha y+\beta z)=x(\alpha y+\beta z)$ $=x\{\alpha y+\beta z+[1-(\alpha+\beta)] \theta\}=\alpha x y+\beta x z+[1-(\alpha+\beta)] x \theta=\alpha x y$ $+\beta x z+[1-(\alpha+\beta)] \theta=\alpha x y+\beta x z=\alpha f(y)+\beta f(z)$; i.e., $f$ is actually linear, and consequently is an $n \times n$ matrix with real entries. Thus, $S$ is equivalent to the matrix semigroup $T_{n}$, since the mapping $s \rightarrow s-e \rightarrow l_{s-e}$ is clearly a $1-1$ affine mapping of $S$ onto $T_{n}$. If $S$ has a left zero, the proof proceeds similarly, using right multiplications. 
It perhaps should be remarked that the hypotheses on $S$ in the previous theorem can be changed slightly: one need not require that the zero and unit actually be in $S$, but that there is a zero and/or unit for $M(S)$ somewhere in $M(S)$. In many examples this is actually the case.

2. In this section, characterizations are given of all one dimensional afine (topological or not) semigroups and of all two dimensional compact affine topological semigroups. More precisely, if $S$ is a one dimensional affine semigroup the multiplication is one of four kinds unless $S=M(S)$, in which case a fifth multiplication is possible. If $S$ is a two dimensional compact affine topological semigroup, then the multiplication is one of seventeen kinds. With this information, it is possible to say in most cases that $S$ is equivalent to sub-semigroups of certain fixed semigroups of the real line or of the plane. The methods used here become somewhat cumbersome in dimension three or greater, but it is expected that refinements of the techniques will be successful there, also.

Recall that the kernel $K$ of a compact topological semigroup $S$ is its minimal two-sided ideal and is known to be closed. It is also known to be the union of the minimal left ideals of $S$, and is also the union of the minimal right ideals of $S$. Each minimal left and minimal right ideal is closed and is convex when $S$ is affine.

THEOREM 9. If $S$ is a one dimensional affine semigroup then $M(S)$ is equivalent to the real line under one of the following multiplications: (a) usual, (b) $x y=0$, all $x, y$, (c) $x y=x+y$, all $x, y$, (d) $x y=x$, all $x, y$, (e) $x y=y$, all $x, y$.

Proof. Denote by $M$ the manifold generated by $S$; the first claim is that $M$ contains an idempotent. To see this, fix $p \in M$. If $p^{2} \neq p$, let $p^{3}=\alpha p$ $+(1-\alpha) p^{2}$. If $\alpha=0$, then $\left(p^{2}\right)^{2}=p^{4}=p^{3} \cdot p=p^{2} p=p^{3}=p^{2}$, and $p^{2}$ is the desired idempotent. If $\alpha \neq 0$, direct computation shows that $((\alpha-1) / \alpha) p+(1 / \alpha) p^{2}$ is an idempotent, so that $M$ has an idempotent, say $e$. Now suppose first there exists $p \neq e$ such that $p^{2}=e$. Then $p e=p^{3}=e p$. If $p^{3}=p$, then $p e=p=e p, e$ is a unit and $(p+e) / 2$ is a zero (for $M(S))$, and the mapping $\alpha e+(1-\alpha)(p+e) / 2$ $\rightarrow \alpha$ is an equivalence between $M(S)$ and the real line under the usual multiplication. If $p^{3}=e$, then $p e=e=e p, e$ is a zero for $M(S),[\alpha e+(1-\alpha) p]$ - $[\beta e+(1-\beta) p]=e$, and the mapping $\alpha p+(1-\alpha) e \rightarrow \alpha$ is an equivalence between $M(S)$ and the real line under the multiplication (b). Now $p^{3}=\alpha e$ $+(1-\alpha) p$ for some $\alpha$, so $e=e^{2}=\left(p^{2}\right)^{2}=p^{4}=\alpha e p+(1-\alpha) p^{2}=\alpha e p+(1-\alpha) e$, and $\alpha e=\alpha e p$. If $\alpha=0$, then $p^{3}=p$ and if $\alpha \neq 0, e=e p=p^{3}$. Next, suppose for no $p \neq e$ is it true that $p^{2}=e$. Fix $p \neq e$ and let $p e=\alpha e+(1-\alpha) p$, so that $p e=\alpha e+(1-\alpha) p e$. Then $\alpha p e=\alpha e$, and $p e=p$ or $p e=e$. Similarly, $e p=p$ or $e p=e$, and four cases result. If $p e=p=e p$, then $p^{2}=2 p-e$, for otherwise the point $\alpha /(\alpha-2) e+2 /(2-\alpha) p$ has its square equal to $e$ and is not $e$, where $p^{2}=\alpha p+(1-\alpha) e$. But then $[a p+(1-a) e][b p+(1-b) e]=(a+b) p$ $+[1-(a+b)] e$, and the mapping $a p+(1-a) e \rightarrow a$ is an equivalence between $M$ and the reals under addition. Note that in this case $S=M$, since there 
are no proper convex sub-semigroups of the additive reals. If $p e=p$ and $e p=e$, then $p=p e=p(e p)=(p e) p=p p=p^{2}$, and $[\alpha e+(1-\alpha) p][\beta e+(1-\beta) p]$ $=\alpha e+(1-\alpha) p$. If $p e=e$ and $e p=p$, similar arguments show $[\alpha e+(1-\alpha) p]$ $\cdot[\beta e+(1-\beta) p]=\beta e+(1-\beta) p$. Then the mapping $\alpha e+(1-\alpha) p \rightarrow \alpha$ is an equivalence between $M$ and the reals under either (d) or (e). Finally, if $p e=e$ and $e p=e$, let $p^{2}=\alpha e+(1-\alpha) p$, and $u=(\alpha /(\alpha-1)) e+(-1 /(\alpha-1)) p$ (note that $\alpha \neq 1$ since $\left.p^{2} \neq e\right)$. Then $u^{2}=u \neq e, u$ is a unit for $M$, and the mapping $\beta u+(1-\beta) e \rightarrow \beta$ is an equivalence between $M$ and the reals under usual multiplication.

COROLlaRy. If $S$ is a one dimensional compact affine topological semigroup, then $S$ is equivalent to either (a) an interval $\alpha \leqq x \leqq \beta$, under the usual multiplication of reals, where $-1 \leqq \alpha<\beta \leqq 1$, or (b) the interval $0 \leqq \alpha \leqq 1$, with either the multiplication $\alpha \beta=0, \alpha \beta=\alpha$, or $\alpha \beta=\beta$ all $\alpha, \beta$.

The above theorem and corollary complete the characterization of the one dimensional semigroups. The remainder of this section is devoted to those affine semigroups which are two-dimensional compact and topological, and the general procedure is roughly the same as before, save for considerable complication. The cases considered are (a) there exists a two dimensional minimal left (or minimal right) ideal, (b) there exists a one dimensional minimal left ideal and a one dimensional minimal right ideal, (c) there exists a one dimensional minimal left ideal and all minimal right ideals are degenerate or the dual of this, and (d) $S$ has a zero. It is emphasized that in the rest of this section $S$ is a two dimensional compact affine topological semigroup.

Lemma 1. If $S$ has a 2-dimensional minimal left ideal, then $x y=x$, all $x, y \in M(S)$. Dually, if $S$ has a 2-dimensional minimal right ideal, then $x y=y$, all $x, y \in M(S)$.

Proof. Let $L$ be a two dimensional minimal left ideal. Then there exist $e, f, g$ distinct points of $L$ such that $\{e, f, g\}$ forms an affine basis for $M(S)$; for example, $e \neq f$ can be chosen, and choose $g \in L \backslash M(\{e, f\})$. Recall now that $x \in L$ implies $S x=L$ and $x S x=\{x\}$; these facts imply $x t=x$, all $t \in L$. As a consequence, $(\alpha e+\beta f+\gamma g)(a e+b f+c g)=\alpha e+\beta f+\gamma g$, whenever $\alpha+\beta+\gamma=1$, $a+b+c=1$; i.e., multiplication is $x y=x$. A similar argument applies if $S$ has a 2-dimensional minimal right ideal.

LemMa 2. If $S$ has both a one dimensional minimal left ideal and a one dimensional minimal right ideal, then $M(S)$ is equivalent to the plane, with the multiplication $(x, y)(a, b)=(x, b), S=K$, and $S$ is equivalent to the unit square with vertices $(0,0),(1,0),(1,1)$ and $(0,1)$.

Proof. Let $L$ and $R$ be the minimal left and right ideals respectively. Then (as was seen in proof of Theorem 3) $L \cap R=\{g\}$. Since $L$ and $R$ are bounded closed line segments, $L$ and $R$ have end-points $e$ and $f$ respectively for which 
$g \neq e, g \neq f$. Since $\{g\}=L \cap R=S e \cap f S$, it follows $g=f e$ and $f e=g \notin M(\{e, f\})$ : if $f e=\alpha e+(1-\alpha) f$, then $f e=f^{2} e=\alpha f e+(1-\alpha) f$, so $(1-\alpha) f e=(1-\alpha) f$, and $f e=e$ or $f$, a contradiction. Thus, $\{e, f, f e\}$ form an affine basis for $M(S)$. Note then that $e f=\alpha e+\beta f+\gamma f e$, for some $\alpha, \beta, \gamma$ such that $\alpha+\beta+\gamma=1$, and $e=e f e=\alpha e+\beta f e+\gamma f e$ (by Theorem 3), so that $\alpha=1$ and $\beta+\gamma=0$. Similarly $f=f e f=\alpha f e+\beta f+\gamma f e$ implies $\beta=1$ and $\alpha+\gamma=0$. Together, these imply $e f=e+f-f e$, and then $(\alpha e+\beta f+\gamma f e)(a e+b f+c f e)=\alpha e+b f+[1-(\alpha+b)] f e$, whenever $\alpha+\beta+\gamma=1, a+b+c=1$. It is then easy to see that the mapping $\alpha e+\beta f+\gamma f e \rightarrow(\alpha, \beta)$ is an affine homeomorphism between $M(S)$ and $R^{2}$ which is also an isomorphism between $M(S)$ and $R^{2}$ with the multiplication $(\alpha, \beta)(a, b)=(\alpha, b)$. It is also clear that under this mapping $S$ is equivalent to a compact affine sub-semigroup $T$ of $R^{2}$ containing $(0,1),(0,0),(1,0)$, and $(1,1)$, and by direct computation, $x S x=\{x\}$, all $x \in S$, i.e., $K=S$, by Theorem 3. Now we show $S$ equivalent to the unit square. Let $g$ be an extreme point of $S$, and let $e$ and $f$ be the other end points respectively of the minimal left ideal $S g$ and the minimal right ideal $g S$ (note that both $S g$ and $g S$ are nondegenerate line segments: if $a, b \in K=S$ the map $x a \rightarrow x b$ of $S a$ into $S b$ is onto and also $1-1$, the latter since $x a=y b \Rightarrow x=x a x=y b x \in y S$; but $x \in S y$ also, since $x, y \in S a \Rightarrow S a=S x=S y$ and then $x \in y S \cap S y=\{y\} \Rightarrow x=y$. Hence the cardinal of $S a=$ the cardinal of $S b$ and so $\operatorname{dim} L=1$ implies' $\operatorname{dim} S a$ and $\operatorname{dim} S b \geqq 1$. But there exists no minimal left ideal of $\operatorname{dim} 2$ by the previous lemma, for then the incorrect multiplication obtains. In like fashion, $\operatorname{dim} R$ $=1$, all $R$. Now the claim is made that $e$ and $f$ are extreme points: if $e$ $=\alpha x+(1-\alpha) y$ with $x, y \in S, 0 \leqq \alpha \leqq 1$, then $g=g e=\alpha g x+(1-\alpha) g y$. Since $g$ is an extreme point, $g=g x$ or $g=g y$, and so $x=x g x=x g \in S g$ or $y=y g y=y g$ $\in S g$. If either $x \in S g$ or $y \in S g$, then $e \in[x, y]$ implies both $x, y \in S g$, and $e$ an extreme point of $S g$ implies $e=x$ or $e=y$; i.e., $e$ is an extreme point of $S$. Similarly $f$ is an extreme point. As seen in the proof above $g=f e$, and $e f=e+f$ $-f e$. It is now claimed that $e f$ is an extreme point and the convex hull of $\{e, f, f e, e f\}$ is $S$. If $e f=\alpha x+(1-\alpha) y$, then $f=f e f=\alpha f x+(1-\alpha) f y$, so $f$ an extreme point implies $f=f x$ or $f y$ and $x f=x f x=x$ or $y f=y f y=y$; i.e., $x \in S f$ or $y \in S f$, and so $e f \in S f \cap[x, y]$ implies $x, y \in S f$. Similarly, $e f=\alpha x+(1-\alpha) y$ implies $e=e f e=\alpha x e+(1-\alpha) y e, e=x e$ or $y e, x=x e x=e x$ or $y=y e y=e y$, $x \in e S$ or $y \in e S$, and $x, y \in e S$; i.e., $x, y \in e S \cap S f=\{e f\}$, and $e f$ is an extreme point of $S$. Thus $f e, e, f$, ef are extreme points of $S$. Now let $x \in S=K$, and let $\{a\}=S x \cap f e S,\{b\}=S x \cap e S$. Then $e S \cdot f e=e(S f) e=\{e\}$ implies $b f e=e$ and $f e S \cdot f e=\{f e\}$ implies afe $=f e$. Since $x=\alpha a+(1-\alpha) b$ it follows that $x f e$ $=\alpha f e+(1-\alpha) e$. But then since $f e$ and $e$ are end points of $S f e=S e, 0 \leqq \alpha \leqq 1$, and since $a \in f e S=f S$ and $b \in e S=e f S$, it follows that $x \in$ convex hull of $\{e, f, e f, f e\} ;$; i.e., $S$ is this convex hull. It is now easy to verify that the mapping $\alpha e+\beta f+\gamma f e \rightarrow(\alpha, \beta)$ takes $S$ onto the unit square.

Lemma 3. If $S$ has a one dimensional minimal left ideal and all minimal right ideals are degenerate, then $M(S)$ is equivalent to the plane under one of the 
following multiplications: $(1)(x, y)(a, b)=(x a, x b+y),(2)(x, y)(a, b)=(x a, y)$, or $(3)(x, y)(a, b)=(0, y)$. Dually, if $S$ has a one dimensional minimal right ideal but all minimal left ideals are degenerate, the multiplication is either ( $\left.1^{\prime}\right)$ $(x, y)(a, b)=(x a, y a+b),\left(2^{\prime}\right)(x, y)(a, b)=(x a, b)$, or $\left(3^{\prime}\right)(x, y)(a, b)=(0, b)$.

Proof. Let $L$ be a one dimensional minimal left ideal, $L=[e, f]$, with $e \neq f$. Note that if there exists $u \in M(S) \backslash M(L)$ such that $u^{2}=u$ then $u$ is a left unit for $u \cdot M(L)$ and $u M(L) \subset M(L)$, since $M(L)$ is a left ideal of $M(S)$; then $u \cdot M(L)$ nonvoid implies there exists $k \in M(L) \cdot \ni \cdot u k=k$. Since $e \neq f$, not both $e$ and $f=k$, say $e \neq k$; then $u e=\alpha e+(1-\alpha) k$, so $u e=u^{2} e=\alpha u e+(1-\alpha) k$ and $(1-\alpha) u e=(1-\alpha) k$. If $\alpha \neq 1, u e=k$, so that $u \cdot M(L)=\{k\}$. If $\alpha=1$, $u e=e$. Now $\{u, e, k\}$ is an affine basis for $M(S)$, so $(\alpha u+\beta e+\gamma k)(a u+b e+c k)$ $=\alpha a u^{2}+\beta e+(\gamma+\alpha c) k+\alpha b u e$, always. Here, $u^{2}=u$, and in the first case $u e=\{k\}$, the product is $\alpha a u+\beta e+[1-(\alpha a+\beta)] k$. In the second case $u e=e$, and the product becomes $\alpha a u+(\beta+\alpha b) e+[1-(\alpha a+\beta+\alpha b)] k$. But then the mapping $\alpha u+\beta e+\gamma k \rightarrow(\alpha, \beta)$ is an equivalence between the plane, with either $(x, y)(a, b)=(x a, y)$ or $(x, y)(a, b)=(x a, x b+y)$. If there exists no $u \in M(S) \backslash M(L)$ for which $u^{2}=u$, fix $u \in S \backslash M(L)$; then since $u L \subset L$ and $L$ has the fixed point property, there exists $k \in L$ such that $u k=k$, where it can be assumed $k \neq e$. Now, $u^{2}=\alpha u+\beta k+\gamma e$ implies $k=u^{2} k=\alpha k+\beta k+\gamma e$, so $\gamma k=\gamma e$. But $k \neq e$, so that $\gamma=0$ and $u^{2}=\alpha u+\beta k \in M(\{u, k\})$. Since every minimal right ideal is degenerate, $k$ is a left zero for $M(S)$, and it follows easily that $M(\{u, k\})$ is a semigroup, with $k$ as zero. By assumption the only idempotent in $M(\{u, k\})$ is $k$ itself, and Theorem 9 shows that $u^{2}=k$. Next, note that if $u e=\alpha e+(1-\alpha) k$, then $k=k e=u^{2} e=\alpha u e+(1-\alpha) k$, so $\alpha k=\alpha u e$ and (whether $\alpha=0$ or not) $u e=k$. But then the product becomes $\alpha a k+\beta e$ $+(\gamma+\alpha c) k+\alpha b k=0 \cdot u+\beta e+(1-\beta) k$, and the mapping $\alpha u+\beta e+\gamma k \rightarrow(\alpha, \beta)$ is an equivalence between $M(S)$ and the plane, with $(x, y)(a, b)=(0, y)$. Similar arguments apply when there exists a minimal right ideal of dimension one.

REMARK 1. In the previous lemma the multiplication $(x, y)(a, b)$ $=(x a, x b+y)$ can be obtained as a matrix multiplication, specifically by representing $(x, y)$ as the matrix

$$
\left(\begin{array}{ll}
x & y \\
0 & 1
\end{array}\right)
$$

The following argument shows that any compact affine semigroup relative to this multiplication must be equivalent to a subset of the semigroup $\{(x, y):|x|+|y| \leqq 1\}:$ in the proof of the previous lemma, for the case being considered, $u k=k, u e=e$, so $u$ is a unit; but then $k$ could be chosen as $(e+f) / 2$ (for $u \cdot(e+f) / 2=(e+f) / 2)$. The mapping $F: \alpha u+\beta e+\gamma k \rightarrow(\alpha, \beta)$ then takes $u$ into $(1,0), e$ into $(0,1), k$ into $(0,0)$, and $f$ into $(0,-1)$, and $F(S)=T$ must contain the points $(0,1),(0,0)$, and $(0,-1)$. If $(x, y) \in T$, so also is 
$(x, y)(0,1)=(0, x+y)$ and $(x, y)(0,-1)=(0,-x+y)$, and $|x|+|y|=x+y$, $-x-y, x-y$, or $-x+y$. Now $(0,1)$ and $(0,-1)$ are the end points of $F(L)$ in $T$, so $(0, x+y)=(x, y)(0,1) \in F(L)$ and $(0,-x+y)=(x, y)(0,-1) \in F(L)$ implies $-1 \leqq x+y \leqq 1$ and $-1 \leqq-x+y \leqq 1$. Thus, $x+y \leqq 1,-x-y \leqq 1$, and $|x|+|y| \leqq 1$ if $|x|+|y|=x+y$ or $-x-y$. Similarly, $-x+y \leqq 1$ and $x-y \leqq 1$ implies $|x|+|y| \leqq 1$ when $|x|+|y|=-x+y$ or $x-y$; i.e., in all cases, $|x|+|y| \leqq 1$.

The compact affine semigroups relative to the multiplications (2) and (3) are even easier to locate. In the case of $(2),(x, y)^{n}=\left(x^{n}, y\right)$, so it is clear that if $F(S)=T$ is to be compact, it must be true that $-1 \leqq x \leqq 1$. Here since $S$ contained $e$, and $k$ could be chosen as $f(u L=\{k\})$ it follows that $(0,1)$ and $(0,-1) \in T$. Then if $(x, y) \in T$, so also is $(0, y)=(x, y)(0,1)$ and $|y| \leqq 1$; i.e., $T \subset$ the square with vertices $(1,1),(1,-1),(-1,-1)$ and $(-1,1)$. In the final multiplication $(3)(0,0),(0,1)$, and $(1,0) \in T$ and since $(x, y)^{2}=(0, y)$, all $(x, y) \in T$ it is clear that $a \leqq y \leqq 1$, where $a$ is the image of $f$ under $F ; a \leqq 0$. In this final case, then, all that can be said of $T$ is that it can be any compact convex set lying between the lines $y=1$ and $y=a, a \leqq 0$, and containing $(1,0)$, $(0,1),(0,0)$, and $(0, a)$.

Lemma 4. If $S$ has a zero 0 , there exists $x$ in $M(S)$ with $x \neq 0$ and $x^{2}$ $\in M(\{0, x\})$.

Proof. Fix $p \in S, p \neq 0$. If $p^{2} \in M(\{0, p\})$, the lemma is proved. If $p^{2} \notin M(\{0, p\})$, then $\left\{0, p, p^{2}\right\}$ is an affine basis for $M(S)$; let $p^{3}=a p+b p^{2}$ $+c 0$, with $a+b+c=1$. Then $p^{4}=\left(a+b^{2}\right) p^{2}+a b p+(b c+c) 0$. If $a=0$, then $\left(p^{2}\right)^{2}=p^{4}=b^{2} p^{2}+\left(1-b^{2}\right) 0 \in M\left(\left\{0, p^{2}\right\}\right), p^{2} \neq 0$, and the theorem is proved. If $a \neq 0$, let $x=p^{2} / 2 a+(-b / 2 a) p+[1-(1 / 2 a-b / 2 a)] 0$, and it follows that $x^{2}=(x+0) / 2$ and $x \neq 0$.

LEMMA 5. If $S$ has a zero and there exists $n \in M(S)$ such that $n^{2}=0, n \neq 0$, then $M(S)$ is equivalent to the plane relative to one of the following multiplications: (1) $(x, y)(a, b)=(x a, x b+y a),(2)(x, y)(a, b)=(x a, y a)$, or the dual $(x, y)(a, b)$ $=(x a, x b),(3)(x, y)(a, b)=(x a, 0)$, or the dual $(x, y)(a, b)=(0, x a)$, or (4) $(x, y)(a, b)=(0,0)$.

Proof. Observe first that if there exists $p \in M(S)$ such that $n p=n$ (or, dually $p n=n)$, then there exists an idempotent $x \in M(S) \backslash M(\{0, n\})$ such that $n x=n(x n=n):$ if $y \in M\{0, n\})$ then $n y=0=y n$, so that if either $n y=n$ or $y n=n$, then $y \in M(\{0, n\})$; thus, if $p^{2}=p$, $p$ itself is the desired idempotent. If $p^{2} \neq p$, and if there exist $x, y \in M\left(\left\{p, p^{2}\right\}\right)$ such that $x y \in M\left(\left\{p, p^{2}\right\}\right)$, then $n x y=n, n p=n, n p^{2}=n \Rightarrow n \cdot m(S)=0$, since $\left\{p, p^{2}, x y\right\}$ form a basis; in particular $0=n \cdot 0=n$, a contradiction. Thus, $M\left(\left\{p, p^{2}\right\}\right)$ is a semigroup, and it is easy to see that $0 \notin M\left(\left\{p, p^{2}\right\}\right)$. But now Theorem 9 provides the desired $x=x^{2} \in M\left(\left\{p, p^{2}\right\}\right)$. 
Observe next that $n \cdot M(S) \cup M(S) \cdot n \subset M(\{0, n\})$ : if, for example, $n x \notin M(\{0, n\})$ for some $x \in M(S)$, then $\{n x, 0, n\}$ forms an affine basis for $M(S)$ and since $n \cdot n x=n \cdot 0=n \cdot n=0$, it follows that $n \cdot x=0 \in M(\{0, n\})$, a contradiction.

The final preliminary observation is that if $p \notin M(\{0, n\})$ and $n p \neq 0$ then there exists $x \in M(\{0, p\})$ such that $n x=n$ (and dually): if $n p=n$, there is nothing to prove, and if $n p \neq n$, then $n p \in M(\{0, n\})$ by the preceding observation, and there exists $a, b$ such that $a+b=1$ and $n p=a 0+b n$. Let $x=\alpha 0+\beta p$, where $\alpha=1-1 / b, \beta=1 / b$. Then $x \in M(\{0, p\})$ and $n x=n$.

For the proof of the lemma, let $\{0, n, x\}$ be an affine basis for $M(S)$, and note that $(\alpha x+\beta n+\gamma 0)(a x+b n+c 0)=\alpha a x^{2}+\beta a n x+\alpha b x n$ $+(\gamma+\beta b+\alpha c+\beta c) 0$, whenever $\alpha+\beta+\gamma=1, a+b+c=1$. Two cases are encountered.

CASE 1. There exists $x \in M(S)$ such that $n x=n$ or $x n=n$. By the first observation it may be assumed that $x^{2}=x \notin M(\{0, n\})$. Then the product becomes $\alpha a x+\beta a n x+\alpha b x n+(\gamma+\beta b+\alpha c+\beta c) 0$. If $n x=n$, then $x n=\delta 0+\epsilon n$ implies $x n=x^{2} n=\delta 0+\epsilon x n$, so $\delta x n=\delta 0$ and $x n=n$ or 0 . Similarly, if $x n=n$ it follows that $n x=n$ or 0 . Thus one obtains the subcases $n x=n=x n, n x=n$ and $x n=0 ; x n=n$ and $n x=0$. The first gives the product $\alpha a x+(\beta a+\alpha b) n$ $+[1-(\alpha a+\beta a+\alpha b)] 0$, the second gives $\alpha a x+\beta a n+[1-(\alpha a+\beta a)] 0$, and the third gives $\alpha a x+\alpha b n+[1-(\alpha a+\alpha b)] 0$, and it is clear then that the mapping $\alpha x+\beta n+\gamma 0 \rightarrow(\alpha, \beta)$ is an equivalence between $M(S)$ and the plane, the latter relative to either multiplications (1) or (2).

CASE 2. $n x$ is never $n$ and $x n$ is never $n$. In this case, the final observation above shows that $n x=0=x n$, for all $x \in M(S)$. If $M(S) \backslash M(\{0, n\})$ contains an idempotent $x$, then the product becomes $\alpha a x+(1-\alpha a) 0$. If zero is the only idempotent in $M(S)$, two subcases occur: (a) there exists $x \in M(\{0, n\})$ such that $M(\{0, x\})$ is a semigroup, (b) $N(\{0, x\})$ is a semigroup for no $x \notin M(\{0, n\})$. In case (a), it follows from Theorem 9 that $x^{2}=0$, and all products in $M(S)$ are zero. In case (b), note then that $x \in M(\{0, n\})$ implies $x^{2} \neq 0$, for otherwise $M(\{0, x\})$ is a semigroup. Note also that $\left(x^{2}\right)^{2} \in M\left(\left\{0, x^{2}\right\}\right)$ if $x^{2} \notin M(\{0, n\})$, for if $x^{2}=d x+e n+f 0$, then $\left(x^{2}\right)^{2}=d^{2} x^{2}+\left(1-d^{2}\right) 0$. Thus, $x^{2} \in M(\{0, n\})$, and it is clear (since $x^{2} \neq 0$ and $y \in M(\{0, n\})$ implies $y^{2}=0$ ) that it may be assumed $x^{2}=n$; the product is then $0 x+\alpha a n+(1-\alpha a) 0$. Thus, in Case 2, the mapping $\alpha x+\beta n+\gamma 0 \rightarrow(\alpha, \beta)$ is an equivalence between $M(S)$ and the plane, relative to one of the multiplications (3) or (4), and the proof is concluded.

REMARK 2. It is clear that any subset of the plane containing $(0,0)$ can be a semigroup relative to multiplication (4). It is also obvious that if $T$ is a compact semigroup relative to any of the remaining multiplications, then $T \subset\{(x, y):|x| \leqq 1\}$, for $(x, y)^{n} \rightarrow \infty$ otherwise. For the multiplication (1) it is easy to see that $T$ meets each of the lines $x= \pm 1$ in at most the points $(1,0)$ and $(-1,0)$; for example, if $(1, y) \in T$, then $(1, y)^{n}=(1, n y) \rightarrow(1, \infty)$ unless 
$y=0$, and similarly for $(-1, y)$. In this case it can also be proved there exists $K>0$ such that $T \subset\{(x, y): K|x|+|y| \leqq K\} ;$ it is easy to see that this latter set is a semigroup. To show the above containment, note first that $T$ can be assumed to contain the unit $(1,0)$, for the convex hull $T^{\prime}$ of $T \cup\{(1,0)\}$ is a compact convex semigroup. Further, it can be assumed that $T$ contains $\delta=(-1,0)$ and contains $(-x,-y)$ whenever it contains $(x, y)$, for the convex hull of $T^{\prime} \cup \delta T^{\prime}$ is such a semigroup containing $T$. Now for $n=0,1,2,3, \cdots$, let $x_{n}=1 / 2^{1 / 2^{n}}$ and $y_{n}=\max \left\{y:\left(x_{n}, y\right) \in T\right\}$. Then, since $\left(x_{n-1}, 2 x_{n} y_{n}\right)$ $=\left(x_{n}, y_{n}\right)^{2} \in T$, it is clear that $2 x_{n} y_{n} \leqq y_{n-1}$, and by induction, that

$$
\frac{y_{n}}{1-x_{n}} \leqq 2 y_{0}\left\{\frac{1}{2^{n}\left\{2^{1 / 2^{n}}-1\right\}}\right\} \rightarrow \frac{2 y_{0}}{\ln 2} ;
$$

hence $y_{n} /\left(1-x_{n}\right) \leqq 2 y_{0} / \ln 2$ for all but finitely many $n$ and there exists $K>0$ such that $y_{n} /\left(1-x_{n}\right) \leqq K$, all $n$. If $(x, y)$ is in $T$ and $K x+y>K$, there exists $n$ such that $x<x_{n}$, and then $K<y /(1-x)<y_{n} /\left(1-x_{n}\right) \leqq K$, a contradiction. In similar fashion, with $x_{n}$ as above and $y_{n}=\min \left\{y:\left(x_{n}, y\right) \in T\right\}$, one obtains

$$
\frac{y_{n}}{1-x_{n}} \geqq 2 y_{0} \frac{1}{2^{n}\left\{2^{1 / 2^{n}}-1\right\}} \rightarrow \frac{2 y_{0}}{\ln 2}
$$

so there exists $K^{\prime}<0$ such that $y /(1-x) \geqq K^{\prime}$ and so $K^{\prime} x+y \geqq K^{\prime}$, all $(x, y) \in T$. At this point, it may be assumed $K x+y \leqq K$ and $K x-y \leqq K$, all $(x, y) \in T$. But $T$ is symmetric, so if $(x, y)$ is in Quadrant II, $(-x,-y) \in T$ and so $K(-x)-(-y) \leqq K$; i.e., $-K x+y \leqq K$. Similarly, $-K x-y \leqq K$, so for all $(x, y) \in T, K|x|+|y| \leqq K$.

A final remark relative to the multiplications of Lemma 5 is this: any convex symmetric set $T$ containing $(0,0)$ is a semigroup relative to multiplications (2), for if $(x, y),(a, b) \in T$ then $(x, y)(a, b)=(x a, y a)=a(x, y)$ $=a(x, y)+(1-a)(0,0) \in T$ if $a \geqq 0$ and $(x, y)(-a,-b) \in T$ if $a \leqq 0$. But then $-[(x, y)(-a,-b)]=-[(-x a,-y a)]=(x a, y a)=(x, y)(a, b) \in T$, by symmetry.

Lemma 6. If $S$ has a zero 0 and $n^{2}=0$ for no $n \neq 0$ in $M(S)$, then $M(S)$ is equivalent to the plane relative to either the multiplication $(1)(x, y)(a, b)=(x a, y b)$ or $(2)(x, y)(a, b)=(x a-y b, x b+y a)$.

Proof. By Lemma 4, there is $x \in M(S)$ for which $x \neq 0$ and $M(\{0, x\})$ is a semigroup, and by Theorem 9 there is an idempotent $e \in M(\{0, x\})$ with $e \neq 0$ (since by assumption $\left.x^{2} \neq 0\right)$. Fix $p \notin M(\{0, e\})$. It is then clear that either $e p \notin M(\{0, e\})$ or $e p \in M(\{0, e\})$ and also either $p e \notin M(\{0, e\})$ or $p e \in M(\{0, e\})$. Note that if $e p=p$, then $e p \notin M(\{0, e\})$, since $p \notin M(\{0, e\})$. Conversely, if $e p \in M(\{0, e\}),\{0, e, e p\}$ forms an affine basis for $M(S)$, and then $e \cdot 0=0, e \cdot e=e, e \cdot e p=e p$ implies $e$ is a left unit for $M(S)$ and consequently $e p=p$. Thus, $e p=p$ if and only if $e p \notin M(\{0, e\})$, if and only if $e$ is a 
left unit; similarly, $p e=p$ if and only if $p e \in M(\{0, e\})$ if and only if $e$ is a right unit. There are now four cases to consider: (1) $e p$ and $p e \in M(\{0, e\})$, (2) $e$ is a left unit and $p e \in M(\{0, e\}),(3) e$ is a right unit and $e p \in M(\{0, e\})$, and (4) $e$ is a unit.

CASE 1. $e p$ and $p e \in M(\{0, e\})$. If $p e=\alpha e+(1-\alpha) 0$, then $e p e=\alpha e+(1-\alpha) 0$ $=p e$, and similarly $e p e=e p$; i.e., $p e=e p$. But then, since $\{0, e, p\}$ forms an affine basis for $M(S)$, it is clear that $M(S)$ is abelian. Now $e \cdot 0=0$ and $e p \in M(\{0, e\})$ so there exists $q \in M(\{0, p\})$ such that $e q=e=q e$ (if $e p=0$, then $e \cdot e=e, e p=0$ imply $e \cdot(p+e) / 2 \in M(\{0, e\})$ and $e \cdot(p+e) / 2 \neq 0)$. Thus, it can be assumed that $e p=e=p e$. Further, $p^{2} \in M(\{e, p\})$, for $\left\{p, p^{2}, e\right\}$ form an affine basis otherwise, and $e \cdot e=e, e p=e, e p^{2}=e$ would imply $e=0$. Thus $M(\{e, p\})$ is a semigroup and if $n=e-p+0$, then $n^{2}=0-e+p^{2}$, so the assumption that no $n^{2}=0$ save $n=0$ implies $p^{2} \neq e$. If $p^{2}=p$ there is an idempotent $u$ in $M(\{e, p\})$ different from $e$. If $p^{2} \neq p$, let $p^{2}=\alpha e+(1-\alpha) p(\alpha \neq 0, \alpha \neq 1)$ and let $u=\alpha /(\alpha-1) e+(-1 /(\alpha-1)) p$; then $u^{2}=u \neq e$. In either case, Theorem 9 implies the idempotent $u$ is a unit for $M(\{e, p\})$, since $e$ is a zero. Now let $f=u-e+0$; then $f^{2}=f, f \neq e, u$, or 0 , and $e f=f e=0$. Then $\{f, e, 0\}$ forms an affine basis for $M(S)$, and $(\alpha f+\beta e+\gamma 0)(a f+b e+c 0)=\alpha a f+\beta b e$ $+[1-(\alpha a+\beta b)] 0$, and the mapping $\alpha f+\beta e+\gamma 0 \rightarrow(\alpha, \beta)$ is an equivalence between $M(S)$ and the plane with multiplication (1).

CASE 2. $e$ is a left unit, $p e \in M(\{0, e\})$. Since $e \cdot e=e$ and $p \cdot e \in M(\{0, e\})$, there exists $q \neq 0$ such that $q \in M(\{e, p\})$ and $q e=e$, so it can be assumed $p e=e$. But then, if $p^{2} \notin M(\{e, p\}),\left\{p, p^{2}, e\right\}$ forms an affine basis, $e \cdot e=e$, $p \cdot e=e, p^{2} \cdot e=e$ imply $e$ is a right zero for $M(S)$, so $0=0 \cdot e=e$, a contradiction. Thus, $M(\{e, p\})$ is a semigroup with $e$ as left unit and $e$ as a right zero. In this case, $(-e+p+0)^{2}=-p+p^{2}+0$, so if $p^{2}=p$, then $-e+p+0$ is an $n \neq 0$ such that $n^{2}=0$; thus $p^{2} \neq p$. But now it follows easily from Theorem 9 that $M(\{e, p\})$ cannot be a semigroup; thus, Case 2 cannot hold, and Case 3 is treated similarly.

CASE 4. $e$ is a unit for $M(S)$. In this case relabel $e$ as $u$ and then there are two subcases: (1) there exists $e=e^{2} \in M(S)$ with $e \neq u, e \neq 0$, (2) there exists no idempotent in $M(S)$ save $u$ and 0 . To handle subcase (1), note that if $f=u-e+0$, then as in Case 1 above, $f$ is another idempotent and the procedure here is then identical with that of Case 1 . In subcase (2), fix $p \notin M(\{0, u\})$ and let $p^{2}=a u+b p+c 0$, with $a+b+c=1$; the claim is that $b^{2}+4 a<0$. First $b^{2}+4 a \neq 0$, for in this case $q=-b u / 2+p+b 0 / 2$ is such that $q^{2}=0$ and $q \neq 0$. Similarly, $b^{2}+4 a$ is not $>0$, for if it were, let $\Delta_{1}=\left(b^{2}+4 a\right)^{1 / 2}$ and let

$$
e=\frac{\Delta_{1}+b}{2 \Delta_{1}} u-\frac{1}{\Delta_{1}} p+\left[1-\left(\frac{\Delta_{1}+b}{2 \Delta_{1}}-\frac{1}{\Delta_{1}}\right)\right] 0 ;
$$

then $e \neq 0, e \neq u$, and $e^{2}=e$. Thus $\Delta=\left(-\left(b^{2}+4 a\right)\right)^{1 / 2}$ is a positive real number, and $q=-(b / \Delta) u+(2 / \Delta) p+[1-(-b / \Delta+2 / \Delta)] 0$ has the property that $q^{2}=2 \cdot 0-u$ and $q \notin M(\{0, u\})$. Then $\{q, u, 0\}$ forms an affine basis, and 
$(\alpha u+\beta q+\gamma 0)(a u+b q+c 0)=(\alpha a-\beta b) u+(\beta a+\alpha b) q+[1-(\alpha a-\beta b+\beta a+\alpha b)] 0$, and the mapping $\alpha u+\beta q+\gamma 0 \rightarrow(\alpha, \beta)$ is an equivalence between $M(S)$ and the plane under the complex multiplication (2).

REMARK 3. The compact affine semigroups $T$ of the plane relative to the last two multiplications are easy to discover. In the case of the multiplication $(x, y)(a, b)=(x a, y b)$, it is easy to see that $T \subset\{(x, y):|x| \leqq 1,|y| \leqq 1\}$, for otherwise $(x, y)^{n}=\left(x^{n}, y^{n}\right) \rightarrow \infty$. Similarly, if $T$ is a compact semigroup relative to complex multiplication it must be true that $T \subset\left\{(x, y): x^{2}+y^{2} \leqq 1\right\}$.

3 . In this section the convexity of the kernel is investigated. It can be readily checked that in each of the semigroups described in $\$ 2$ the kernel is convex; hence, there results

THEOREM 10. If $K$ is the kernel of a compact affine semigroup $S$ of dimension $\leqq 2$, then $K$ is convex.

Note, in fact, that if $\operatorname{dim} S<2$, the theorem has been verified even when $S$ is not compact. In addition, the following theorem sheds some light on the three dimensional case.

THEOREM 11. If $K$ is the kernel of a compact affine topological semigroup $S$ of dimension 3, and if $S$ has an identity element $u$, then $K$ is convex.

Proof. Let $e, f \in K$ and suppose that $m=\alpha e+(1-\alpha) f$ is not in $K$, for some $\alpha$, with $0<\alpha<1$. It can be verified by a straightforward computation that $m e m=m^{2}=m f m$, so that in particular $m^{2} \in K$. Moreover, a similar computation shows that $m^{3}=m^{2} m=(m e m) m=m(e m) m=m e m$, the latter since $e f m$ $=e m$. Thus, $m^{3}=m^{2}$.

CASE 1. Suppose $m^{2} \in M(\{u, m\})$. Then, clearly $M(\{u, m\})$ is a one dimensional subsemigroup with identity element $u$ and zero $m^{2}$. By Theorem 9 of $\$ 2 M(\{u, m\})$ is equivalent to the reals under ordinary multiplication and thus $m$ must equal $m^{2}$.

CASE 2. If $m^{2} \notin M(\{u, m\})$, let $\pi=M\left(\left\{u, m, m^{2}\right\}\right)$. Clearly $\pi$ is a two dimensional subsemigroup with $m^{2}$ as its zero element. If $k \in \pi \cap K$, then $k=k m^{2} k=m^{2}$ (since $m^{2}$ is the zero for $\pi$ ); so $\pi \cap K=\left\{m^{2}\right\}$. Now since $\pi$ disconnects $K$ ( $e$ and $f$ are in different components of $M(S) \backslash \pi$ ), it has been shown that $m^{2}$ is a cut point of $K$. A theorem of Faucett [2] says that under these circumstances $K$ must consist of left or right zeroes, and thus must be a minimal left or right ideal, and hence by Theorem 3 of $\$ 1$ is convex.

Two counter-examples are now offered to possible extensions of the theorem.

Example 1. Let $S$ be the unit cube in $R^{3}$ (i.e., $\{(x, y, z)$ :

$$
\begin{aligned}
& 0 \leqq x \leqq 1 \\
& 0 \leqq y \leqq 1 \\
& 0 \leqq z \leqq 1\})
\end{aligned}
$$


with the multiplication $(a, b, c) \circ(x, y, z)=(a, y, a y)$. It is easy to verify that $S$ is a compact affine topological semigroup without unit. In particular note that this multiplication may be obtained as a matrix multiplication by representing the point $(x, y, z)$ as the matrix

$$
\left(\begin{array}{lll}
1 & y & 0 \\
0 & 0 & 0 \\
x & z & 0
\end{array}\right) .
$$

It is also easy to see that the kernel of $S$ consists of those points in $S$ for which $z=x y$. Thus the kernel is a portion of a hyperbolic paraboloid and definitely not convex. In particular the midpoint of the kernel elements $(0,0,0)$ and $(1,1,1)$ is not in the kernel.

EXAMPLE 2. Let $u, e, f, g$, and $h$ be respectively the points $(0,0,0,1)$, $(1,-1,-1,0),(-1,1,-1,0),(-1,-1,1,0)$ and $(1,1,1,0)$ in $R^{4}$. Let $S$ be the convex hull of these five points and define a multiplication "o" in $S$ by $\left(x_{1}, y_{1}, z_{1}, w_{1}\right) \circ\left(x_{2}, y_{2}, z_{2}, w_{2}\right)=\left(w_{2} x_{1}+x_{2}, w_{1} y_{2}+y_{1}, z_{1} w_{2}+w_{1} z_{2}+y_{1} x_{2}, w_{1} w_{2}\right)$. This again may be considered as a matrix multiplication by representing $(x, y, z, w)$ as the matrix

$$
\left(\begin{array}{lll}
w & 0 & 0 \\
z & w & y \\
x & 0 & 1
\end{array}\right) .
$$

It is easy to check that $S$ is a compact affine topological semigroup with $u$ as its unit, and that $K$ the kernel, consists of those points of $S$ for which $w=0$, and $z=x y$. Thus $e$ and $f$ are kernel elements but their midpoint is not.

At this point the process of classifying three dimensional compact affine topological semigroups might well begin. We do not attempt such a classification but, at the suggestion of the referee, list several pertinent observations. In what follows $S$ is a three dimensional compact affine topological semigroup with kernel $K$.

If $K$ is three dimensional and convex then $K=S$. The structure of $K$ in this case is easily seen to be one of two types.

I. $K$ is any convex body whose points are all left (or right) zeroes; i.e. the minimal right (left) ideals are the points in $K$, and $K$ itself is the minimal left (right) ideal.

II. $K$ is a solid convex cylinder (not necessarily right circular) whose sections parallel to the bases are minimal left (or right) ideals and whose generators are minimal right (left) ideals.

If $S$ has an identity element, $K$ is at most two dimensional. If $K$ is two dimensional, $K$ is a plane section of $S$ and again either the minimal right or left ideals are degenerate or not. In the latter case $K$ must be a parallelogram 
with its interior, and the minimal left ideals are the lines parallel to one pair of edges while the minimal right ideals are the lines parallel to the other pair of edges. If $K$ is one dimensional, it is a line whose points are all either left or right zeroes. Finally, if $K$ is zero dimensional, it is a point and hence a zero.

\section{BIBLIOGRAPHY}

1. A. H. Clifford, Semigroups containing minimal ideals, Amer. J. Math. vol. 70 (1948) pp. 521-526.

2. W. M. Faucett, Topological semigroups and continua with cut points, Proc. Amer. Math. Soc. vol. 6 (1955) pp. 748-756.

3. J. L. Kelley, Convergence in topology, Duke Math. J. vol. 17 (1950) pp. 277-283.

4. M. Krein and D. Milman, On extreme points of regular convex sets, Studia Math. vol. 9 (1940) pp. 133-137.

5. H. Nikaido, $A$ proof of the invariant-mean value theorem on almost periodic functions, Proc. Amer. Math. Soc. vol. 6 (1955) pp. 361-363.

6. J. E. L. Peck, An ergodic theorem for a noncommutative semigroup of linear operators, Proc. Amer. Math. Soc. vol. 2 (1951) pp. 414-421.

7. A. D. Wallace, $A$ note on mobs, I. An. Acad. Brasil Ci. vol. 24 (1952) pp. 329-334.

8. - The structure of topological semigroups, Bull. Amer. Math. Soc. vol. 61 (1955) pp. 95-112.

9. J. G. Wendel, Haar measure and the semigroup of measures on a compact group, Proc. Amer. Math. Soc. vol. 5 (1954) pp. 923-929.

Louisiana State University, Baton Rouge, LA. 